\title{
On the regularity criteria for liquid crystal flows involving the gradient of one velocity component
}

\author{
Helin Guo ${ }^{1}$ \\ ${ }^{1}$ Wuhan University of Technology
}

May 11, 2020

\begin{abstract}
In this paper, we show a regularity criteria for three dimensional nematic liquid crystal flows. More precisely, we prove that the strong solution $\$(\mathrm{u}, \mathrm{d}) \$$ can be extended beyond $\$ \mathrm{~T} \$$, provided $\$ \backslash$ nabla $\mathrm{u}_{-}\{3\} \backslash \operatorname{in}\left\{\mathrm{L}^{\wedge}\{\mathrm{p}\}\left(0, \mathrm{~T} ; \mathrm{L}^{\wedge}\{\mathrm{q}\}\left(\backslash \operatorname{mathbb}\{\mathrm{R}\}^{\wedge}\{3\}\right)\right)\right\} \$$ $\$ \backslash \operatorname{frac}\{2\}\{\mathrm{p}\}+\backslash \operatorname{frac}\{3\}\{\mathrm{q}\} \backslash \operatorname{leq}\{\backslash \operatorname{frac}\{19\}\{12\}+\backslash \operatorname{frac}\{1\}\{2 \mathrm{q}\}\}(\backslash \operatorname{frac}\{30\}\{19\} 3) \$$ with some conditions about the orientation field $\$ \backslash$ nabla_ $\{\mathrm{h}\} \mathrm{d} \backslash \operatorname{in}\left\{\mathrm{L}^{\wedge}\{\backslash\right.$ alpha $\}\left(0, \mathrm{~T} ; \mathrm{L}^{\wedge}\{\backslash\right.$ beta $\left.\left.\}\left(\backslash \operatorname{mathbb}\{\mathrm{R}\}^{\wedge}\{3\}\right)\right)\right\} \$, \$ \backslash$ frac $\{2\}\{\backslash$ alpha $\}+\backslash$ frac $\{3\}\{\backslash$ beta $\} \backslash$ leq $\{\backslash$ frac $\{3\}\{4\}+\backslash$ frac $\{1\}\{2 \backslash$ beta $\}$

\section{Hosted file}

Guo20200505.pdf available at https://authorea.com/users/320247/articles/449840-on-theregularity-criteria-for-liquid-crystal-flows-involving-the-gradient-of-one-velocitycomponent
\end{abstract}

